Research Article

\title{
Invasion of Epithelial Cells Is Correlated with Secretion of Biosurfactant via the Type 3 Secretion System (T3SS) of Shigella flexneri
}

\author{
Duchel Jeanedvi Kinouani Kinavouidi, ${ }^{1,2}$ Christian Aimé Kayath (D), \\ and Etienne Nguimbi ${ }^{1,2}$ \\ ${ }^{1}$ Laboratoire de Biologie Cellulaire et Moléculaire (BCM), Faculté des Sciences et Techniques, Université Marien N'gouabi, BP. 69, \\ Brazzaville, Congo \\ ${ }^{2}$ Institut National de Recherche en Sciences Exactes et Naturelles (IRSEN), Avenue de l'Auberge Gascogne, B.P 2400, \\ Brazzaville, Congo
}

Correspondence should be addressed to Christian Aimé Kayath; chriskayath@yahoo.fr

Received 18 March 2020; Accepted 30 June 2020; Published 29 July 2020

Academic Editor: Patrizia Messi

Copyright (c) 2020 Duchel Jeanedvi Kinouani Kinavouidi et al. This is an open access article distributed under the Creative Commons Attribution License, which permits unrestricted use, distribution, and reproduction in any medium, provided the original work is properly cited.

\begin{abstract}
Biosurfactants are amphipathic molecules produced by many microorganisms, usually bacteria, fungi, and yeasts. They possess the property of reducing the tension of the membrane interfaces. No studies have been conducted on Shigella species showing the role of biosurfactant-like molecules (BLM) in pathogenicity. The aim of this study is to assess the ability of Shigella environmental and clinical strains to produce BLM and investigate the involvement of biosurfactants in pathogenicity. Our study has shown that BLM are secreted in the extracellular medium with EI24 ranging from $80 \%$ to $100 \%$. The secretion is depending on the type III secretion system (T3SS). Moreover, our results have shown that S. flexneri, S. boydii, and S. sonnei are able to interact with hydrophobic areas with $17.64 \%, 21.42 \%$, and $22.22 \%$ hydrophobicity, respectively. BLM secretion is totally prevented due to inhibition of T3SS by $100 \mathrm{mM}$ benzoic and $1.5 \mathrm{mg} / \mathrm{ml}$ salicylic acids. P. aeruginosa harboring T3SS is able to produce $100 \%$ of BLM in the presence or in the absence of both T3SS inhibitors. The secreted BLM are extractable with an organic solvent such as chloroform, and this could entirely be considered a lipopeptide or polypeptide compound. Secretion of BLM allows some Shigella strains to induce multicellular phenomena like "swarming."
\end{abstract}

\section{Introduction}

The ingestion of pathogenic and virulent microorganisms generally affects peoples in both developed and developing countries. Shigella is one of the Gram-negative bacterium belonging to Enterobacteriaceae family and is a causative agent of bacillary dysentery or shigellosis [1]. Children under five years are the most affected. More and more shigellosis is considered like neglected disease; meanwhile, 164300 of death per years have been notified all over the world in 2010 . Most deaths occur in sub-Saharan Africa and in south Asia $[2,3]$. This includes Republic of Congo, and surprisingly, no epidemiological studies have been conducted in this field.
The genus Shigella includes four species (S. flexneri, S. sonnei, S. dysenteriae, and S. boydii). Ten bacteria of S. dysenteriae type 1, and 100 to 180 bacteria of S. flexneri or S. sonnei are enough to produce symptomatic infection.

Shigella's pathogenicity is based on a virulence plasmid pWR100 in which the mxi-spa locus encodes the type three secretion system (T3SS) involved in effector production like IpaB, C, and D (translocator and tip) to invade host cell [4]. A previous study in our laboratory showed that Shigella sp. isolated from Brazzaville wastewater were able to emulsify hydrocarbon from gasoline and/or diesel fuel [5]. According to amphipathic features, biosurfactants display a variety of surface activities, which explain their application in several 
fields related with emulsification, foaming, detergency, wetting, dispersion, pathogenicity, and solubilisation of hydrophobic compounds $[6,7]$. Biosurfactants are produced from a couple of Gram negative bacteria like Pseudomonas aeruginosa and Acinetobacter calcoaceticus. Rhamnolipids are well known among biosurfactant, and many informations have been documented in terms of biochemical and biotechnological applications as well [8].

Shigella pathogenicity mechanisms have been mostly studied using S. flexneri 5a M90T strain as a Gram-negative bacterium model. In this way, this work aims to study the involvement of BLM via the Type Three Secretion System (T3SS) pathways. In addition, this work will assess the approvals that Shigella could use the BLM to promote the invasion and the dissemination inside epithelial cells.

\section{Materials and Methods}

2.1. Strains and Culture Conditions. Four Shigella strains were kindly provided and collected from laboratory of Molecular Bacteriology (Faculté de Médecine, Erasme Campus, Free University of Brussels). These included $S$. flexneri5a M90T, S. flexneri spa40-, S. sonnei, and S. boydii. Three pure culture strains were isolated from patients in Brazzaville University and Hospital Center (CHU-B) in 2018. These were provided by the Virology and Bacteriology Laboratory of this afore hospital. Thirty Shigella sp. strains were isolated in environmental wastewater of Brazzaville using decimal dilution in SS medium. Lab strains like $P$. aeruginosa and E. coli Top10 were used as controls in this study. The strains were spread on the plates containing LB medium with Congo red with $100 \mu \mathrm{g} / \mathrm{mL}$ streptomycin for 24 hours at $37^{\circ} \mathrm{C}$ for wild type and $50 \mu \mathrm{g} / \mathrm{mL}$ for $s p a 40$ mutant.

2.2. Emulsification Index (EI24) Assay. From $5 \mathrm{~mL}$ of bacterial overnight culture, the emulsification index (EI24) was calculated as an indicator for BLM production as previously demonstrated [5]. The medium was adjusted to $\mathrm{pH} 7.2$ and supplemented with gasoline or diesel fuel $(1 \mathrm{~mL}$ for $300 \mathrm{~mL}$ of medium). The EI24 was investigated by adding fuels with LB medium in $1: 1$ ratio $(\mathrm{v} / \mathrm{v})$. The solution was vortexed for $5 \mathrm{~min}$ and incubated for $24 \mathrm{~h}$ at $37^{\circ} \mathrm{C}$. The emulsification rate was calculated through the height of the emulsion layer. In addition, EI24 was determined for gasoline and diesel fuel hydrocarbons. All the experiments were performed in triplicate; EI24 = height of emulsion layer/total height of solution $\times 100$.

2.3. Bacterial Swarming Assays. Swarming was studied for all Shigella strain used in this study, using plate assays containing $0.5 \%$ noble agar and LB medium with $0.5 \%$ dextrose. The mixture was sterilized at $121^{\circ} \mathrm{C}$, during $15 \mathrm{~min}$. After sterilization, the medium was supplemented with adequate antibiotics including streptomycin $100 \mu \mathrm{g} / \mathrm{mL}$ for wild type and kanamycin $50 \mu \mathrm{g} / \mathrm{mL}$ for the Shigella flexneri spa40 mutant. Approximately $6 \mathrm{~h}$ after pouring the plates, bacteria were inoculated and spread by using a sterilized platinum wire with log-phase cells ((OD600) 0.6) grown in their respective media used for the swarming experiments. Swarming plates that were imaged only for their comparative endpoint swarming development were incubated at $30^{\circ} \mathrm{C}$ for $24 \mathrm{~h}$ prior to imaging.

2.4. Bacterial Adhesion Assay. The adhesion of bacteria to hydrophobic surface was evaluated according to the method described by Rosenberg [9]. The hydrophobicity was evaluated according to the following formula: $\% \mathrm{H}=A 0-A$ / $A 0 * 100$ with $A_{0}$ : OD before the mix and $A$ : OD after vortexing of aqueous phase.

2.5. Induction Assay Using Congo Red. Shigella sp. have been cultivated in $5 \mathrm{~mL}$ of the final volume. One $\mathrm{mL}$ of overnight culture was centrifuged and $500 \mu \mathrm{L}$ of sterile PBS and $10 \mu \mathrm{l}$ of Congo red $(10 \mathrm{mg} / \mathrm{ml})$ have been gently added and mixed with the pellet by avoiding breakage of the cells. Samples were incubated at $37^{\circ} \mathrm{C}$ with stirring. After 30 minutes of incubation, samples were centrifuged at $15.000 \mathrm{rpm}$ for 15 minutes at room temperature. Supernatants were gently recovered and mixed with gasoline or diesel fuels. The emulsification index (EI24) has been determined as discribed in the afore section 2.2.

2.6. Extraction of Biosurfactant-like Molecule. Three methods have been used to extract the biosurfactant.

2.6.1. HCL and Ethanol Precipitation. An overnight culture has been centrifuged at $13,000 \times \mathrm{g}$ for 15 minutes. Once the supernatant was collected, $\mathrm{HCl} 1 \mathrm{~N}$ and 90-degree ethanol were added to the supernatant. Precipitates have been generated by incubating samples at $4^{\circ} \mathrm{C}$ in overnight. Mixtures were centrifuged at $13000 \mathrm{~g}$ for 15 minutes to obtain granules. The granules obtained were tested with EI24 to evaluate the ability to emulsify the hydrocarbons.

2.6.2. Ammonium Sulfate Precipitation Test. An overnight culture has been centrifuged at 13,000 rpm for 15 minutes to separate supernatant and pellet. Then, $15 \mathrm{~mL}$ of supernatant were mixed with ammonium sulfate $(80 \%)$ for 15 minutes. Finally, this has been incubated with shaking overnight. The mixture has been centrifuged at $6000 \mathrm{rpm}$ for 30 minutes at room temperature. Pellets were homogenized using PBS buffer. The emulsification activity has been assessed.

2.6.3. Biosurfactant Extraction Using Chloroform. The $24 \mathrm{~h}$ culture was strictly centrifuged at $15,000 \mathrm{~g}$ for 15 minutes to avoid any residual bacteria. One volume of supernatant was added with an equal volume of chloroform (v/v). The mixture was strongly agitated by a vortex. After centrifugation at $6000 \mathrm{rpm}$ for $10 \mathrm{~min}$, the nonaqueous phase is recovered. The solvent was allowed to evaporate completely only without heating above $40^{\circ} \mathrm{C}$. The residue is dissolved in a PBS buffer. The emulsification activity is tested by mixing with gasoline or diesel fuel in comparison with the 
supernatant at the start point. The emulsification Index (EI24) has been determined.

\subsection{Effect of Benzoic Acid and Salicylic Acid on Biosurfactant} Secretion. Viability of Shigella strains has been first evaluated with different concentrations of benzoic acid and salicylic acid. S. flexneri5a M90T was grown in Luria-Bertani broth (LB) in the presence of various concentrations of benzoic acid $(50 \mathrm{mM}, 100 \mathrm{mM}, 250 \mathrm{mM}$, and $500 \mathrm{mM})$ and salicylic acid $(1.5 \mathrm{mg} / \mathrm{mL}, 3 \mathrm{mg} / \mathrm{mL}, \quad 6.25 \mathrm{mg} / \mathrm{mL}$, and $12.5 \mathrm{mg} / \mathrm{mL}$ ). After that, all Shigella strains were grown in Luria-Bertani broth (LB) added with an adequate concentration of benzoic acid or salicylic acid at $37^{\circ} \mathrm{C}$, during 24 hours. All supernatants were centrifuged and the secretion of biosurfactants was assessed by using emulsification assay (EI24).

2.8. Statistical Analysis. GraphPad Prism 7 and Excel software were used for analysis. The data represent the arithmetical averages of at least three replicates. Data were expressed as mean $\pm \mathrm{SD}$, and Student's test was used to determine statistical differences between strains and $p<0.05$ was considered as significant. Principal component analysis (PCA) was used to investigate possible correlations between Shigella and emulsification index (EI24). Prior to ordination, percentage of emulsification activities data were transformed to better meet the assumptions of normality using $\ln (x+1)$. All analysis was performed using CANOCO (Canonical Community Ordination, version 4.5).

\section{Results}

3.1. Screening for Biosurfactant Production. In order to carry out our research, we first assess if Shigella strains would be able to produce BLM in extracellular medium. Figure 1 shows that environmental strains and clinical strains are able to secrete BLM by showing emulsification percentages ranging between $15 \%$ and $100 \%$ (Figure 1(a)). S. flexneri spa40 mutant was not able to produce BLM compared with Pseudomonas aeruginosa used as positive control. The way of strains to produce BLM is shown in Figure 1(b). All strains are not represented (Figure 1(b)).

EI24 of some strains ranges from $80 \%$ to $100 \%$. These included Shigella flexeneri M90T, Shigella boydii (Sbo), Shigella sonnei (Sso), Shigella sp (Ssp), Ssp2, SE3, SE5, SE9, SE11, SE12, SE13, SE14, SE15, SE16, SE18, SE20, SE21, SE22, SEI24, SE25, SE2626, SE27, SE29, and SE30 (Figure 2). The positive control has been found in this rate. SE1, SE8, SE23, and Ssp 1 are ranging between $40 \%$ and $60 \%$. SE4, SE10, and SE2 8 are between $20 \%$ and $40 \%$. SE17 and SE2 from $60 \%$ to $80 \%$ and Shigella flexneri spa40 mutant (spa40-) are not able to produce biosurfactant, and SE6 is about $17 \%$ ranging from $0 \%$ to $20 \%$ (Figure 2 ).

3.2. Ability of Shigella Strain in the Swarming Test. Swarming is induced by the production of BLM. In order to demonstrate how Shigella could disseminate into epithelial cell, we first investigated if all Shigella strains used in this study were able to swarm by using $0.5 \% \mathrm{LB}$ medium $+0.5 \%$ dextrose. As a result, S. flexneri5a M90T, S. sonnei, and $S$. boydii were able to spread and swarm. spa40- was not able to swarm (Figure 3). Some examples of the swarming profile of some Shigella strains after 24 hours are illustrated. We found that $S$. flexneri spa40- cannot swarm and S. sonnei have a particular swarming profile than other Shigella strains used in this study.

3.3. Bacterial Adhesion to Hydrocarbon (BATH). To highlight the production of BLM by Shigella strains to induce interaction with hydrophobic areas, we performed analysis by evaluation of the ability to interact with hydrocarbon. Figure 4 shows the bacteria adhesion profile of some Shigella strains. Only S. flexneri spa40 mutant does not interact with hydrocarbon area (Figure 4(a)). S. flexneri, S. boydii, and $S$. sonnei are positive with BATH techniques including a percentage of hydrophobicity of $17.64 \%, 21.42 \%$, and $22.22 \%$, respectively (Figure $4(\mathrm{~b})$ ).

3.4. Screening of Biosurfactant Secreted by Shigella sp. To highlight the biochemical specifications of the BLM secreted by Shigella strains used in this study, cultures of Shigella strains with supernatant-emulsified hydrocarbons (gasoline or diesel fuel) have been used to identify the type of biosurfactant-like molecules. Precipitation on hydrochloric acid, ammonium sulphate, and ethanol has been done. All strains showed a precipitate at the bottom of the tube (Figure 5(a)). The emulsification index after precipitation has been carried on EI24. Only the precipitate profile of the S. flexneri spa40- supernatant did not emulsify the gas oil and/or diesel fuel. S. flexneri, S. sonnei, S. boydii, and three Shigella sp. have $100 \%$ of EI24 (Figure 5(b)).

Strains with known hydrocarbon emulsification ability were selected from an organic solvent like chloroform using biosurfactant extraction assay. Biosurfactant could be extracted after evaporation of chloroform at $40^{\circ} \mathrm{C}$ from $\mathrm{S}$. flexneri M90T. Nothing was obtained from spa40-. The extract after evaporation, suspended in PBS, was able to emulsify gasoline or gas oil with $100 \%$ of EI24 (Figure 5(b)).

3.5. BLM Is Secreted by Type Three Secretion System (T3SS). Clinical strains including S. flexneri5a M90T, S. sonnei, S. boydii, three Shigella sp., and 30 environmental strains including SE1 to SE30 were cultivated to induce the secretion of effector on Congo red induction. Shigella species have been found to secrete BLM on Congo red induction conditions with EI24 ranging from $80 \%$ to $100 \%$. The mutant S. flexneri spa40- did not emulsify the gasoline and/or diesel fuel in the presence of Congo induction with $0 \%$ of EI24 (Figure 6(a)). Emulsification index after Congo red type 3 secretion system of Shigella strain appearance are illustrated in Figure 6(b).

3.6. Effect of Benzoic Acid and Salicylic Acid on Biosurfactant Production. All Shigella strains were grown in Luria-Bertani (LB) broth in the presence of random concentrations of 


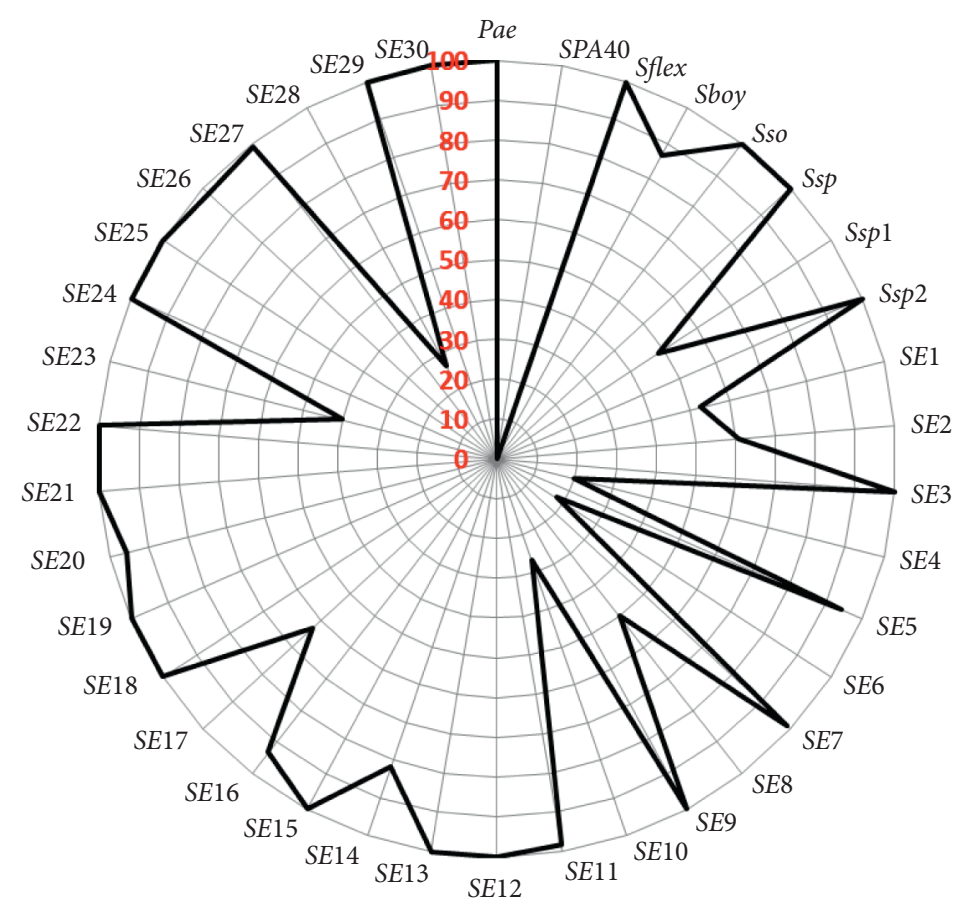

(a)

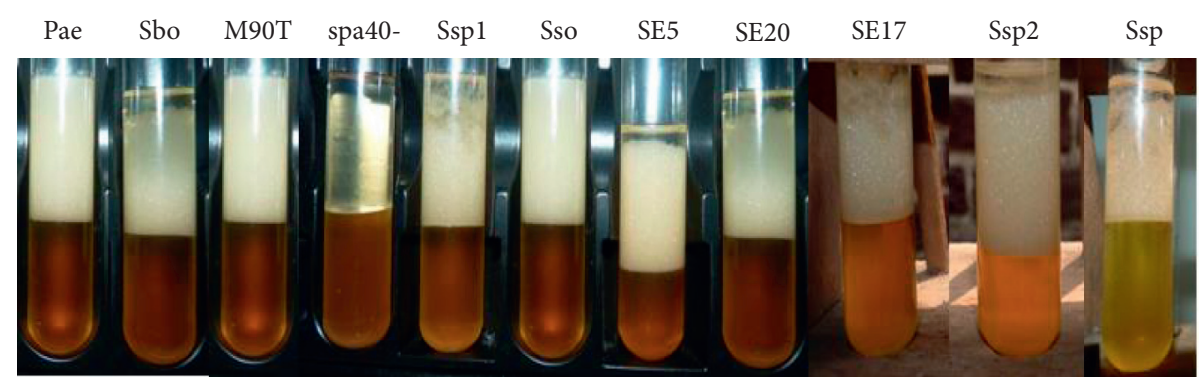

(b)

Figure 1: (a) Emulsification index percentage of all Shigella strains used in this study after 24 hours. Pae: P. aeruginosa used as positive control; M90T: Shigella flexneri 5a strain M90T; spa40-: S. flexneri spa40 mutant; Sbo: S. boydii; Sso: S. sonnei; Ssp, Ssp1, Ssp2: Shigella sp. from clinical strains; SE1 to SE30: Shigella sp. from environmental strains. (b) Emulsification index appearance of some Shigella strains.

benzoic acid and salicylic acid (data not shown). We examined growth at the various concentration of benzoic acid including $50 \mathrm{mM}, 100 \mathrm{mM}, 250 \mathrm{mM}$, and $500 \mathrm{mM}$. As far as salicylic acid is concerned, $1.5 \mathrm{mg} / \mathrm{mL}, 3 \mathrm{mg} / \mathrm{mL}, 6.25 \mathrm{mg} /$ $\mathrm{mL}$, and $12.5 \mathrm{mg} / \mathrm{mL}$ were randomly selected. All Shigella strains grew normally within the physiological range of benzoic acid as determined by CFU per milliliter, but growth was significantly interesting at $100 \mathrm{mM}$ benzoic acid and $1.5 \mathrm{mg} / \mathrm{mL}$ for salicylic acid (data not shown).

To highlight the role of T3SS on the secretion of BLM, we assessed the effect of benzoic and salicylic acids to inhibit the biosurfactant production. Bacteria were previously incubated with $100 \mathrm{mM}$ benzoic acid (BA) and $1.5 \mathrm{mg} / \mathrm{ml}$ salicylic acid (SA), and we showed that S. flexneri M90T, S. sonnei, S. boydii, and SE5 were not able to produce BLM with an emulsification index 0\% EI24 (Figure 7(a)). This easily showed that all Shigella strains do not emulsify anymore gasoline or diesel fuel with benzoic acid or salicylic acid (Figure $7(\mathrm{a})$ ). Strains are able to emulsify gasoline or diesel fuel without benzoic acid or salicylic acid. The appearance is also illustrated (Figure 7(b)). P. aeruginosa has been used as positive control since T3SS is widely conserved in most Gram-negative bacteria, and surprisingly $P$. aeruginosa was able to produce $100 \%$ BLM in the presence or in the absence of BA and SA. It is worth noting that spa40- was not able to produce BLM neither in the presence nor in the absence of BA and SA as previously mentioned (Figure 7(a)7(a)).

\section{Discussion}

This work was conducted with the prime aim of contributing to the understanding of the S. flexneri 5a M90T epithelial cell invasion mechanisms. Shigella strains had been collected from the environmental areas, hospital, or laboratory. All strains had the ability to produce BLM during growth in extracellular medium, and the production is strictly depending on T3SS pathway. This result shows very clearly that these molecules are secreted in the extracellular medium 


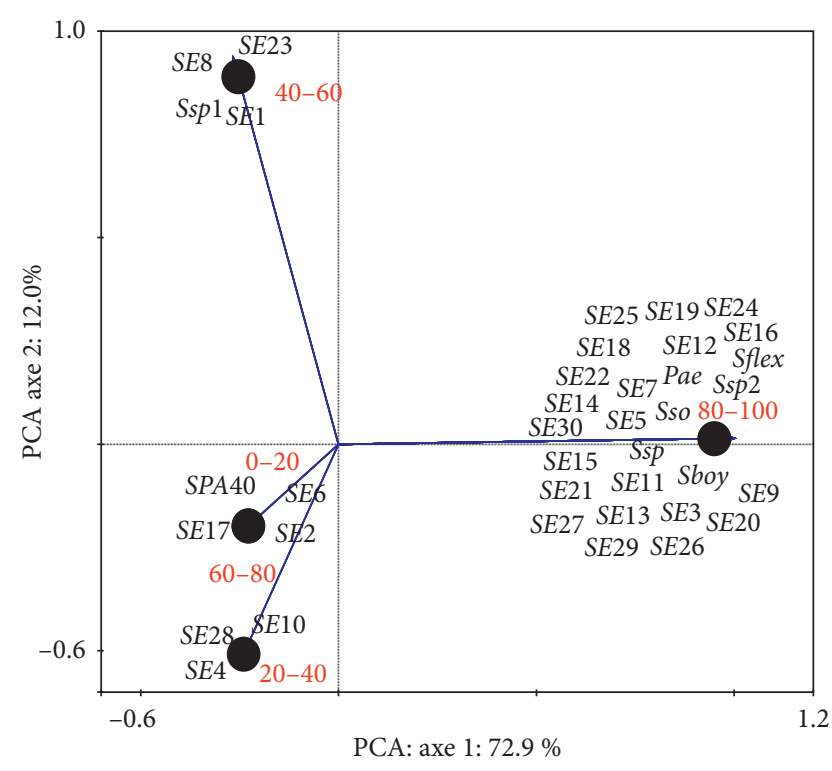

Figure 2: PCA of Shigella strains based on emulsification index (EI24). Pae: P. aeruginosa used as positive control; S. flex: S. flexneri M90T; S. flex-: S. flexneri spa40 mutant; Sboy: S. boydii; Sso: S. sonnei; Ssp 1, 2: Shigella sp. from clinical strains; SE1 to SE30: Shigella sp. from environmental strains.

M90T

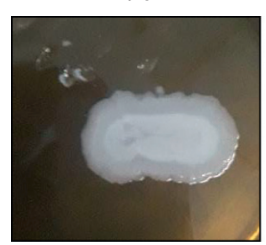

Sbo

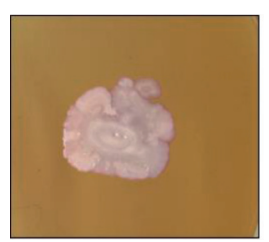

Ssp3

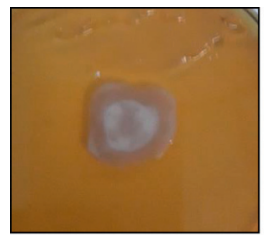

FIGURE 3: Swarming profile of Shigella strains. M90T: Shigella flexneri 5a strain M90T; Sbo: S. boydii; Sso: S. sonnei; spa40-: S. flexneri 5a spa40-; Ssp1, 2, 3: Shigella sp. Pae: $P$. aeruginosa used as positive control and $E$. coli-Top10 used as negative control.

as described by Usman et al. [10]. spa40 mutant which has no T3SS, cannot secrete BLM. Several studies have demonstrated the role of T3SS in the secretion of numbers of effector proteins involved in invasion and dissemination $[11,12]$.
The emulsification index is a direct method for demonstrating the ability of strains to produce biosurfactants or not [5]. Those molecules have been known to form emulsions between two immiscible liquids $[13,14]$. Experiments carried out from the acellular supernatant showed that $S$. flexneri 5a M90T as well as S. boydii, S. sonnei, and other Shigella sp. are able to emulsify gasoline and diesel fuels with EI24 ranging from $80 \%$ to $100 \%$. Gram-negative bacteria are well documented to overcome this phenomenon. These include $P$. aeruginosa [13, 14], Salmonella enteridis [15], Acinetobacter sp. [16], and Serratia Marcescens [17]. Grampositive bacteria are known as being efficient in producing BLM. The spore-forming bacteria like B. subtilis, $B$. lichenifornis, and Lysinibacillus louembei have been widely used to produce BLM [18-20].

Biosurfactants are native of several multicellular phenomena such as swarming described in several bacterial species [21]. By using specific culture media, we have shown that all strains of Shigella genus were positive in the swarming assay. The swarming phenomenon promotes the ability of biosurfactant production. This phenomenon is associated with antibiotic resistance, virulence, and biofilm formation in Proteus mirabilis, Salmonella enterica serovar Typhimurium, and Serratia [22-24]. This idea reinforces the fact that Shigella sp. could also use biosurfactants in its pathogenicity. No genes have been identified to be directly involved in BLM biosynthesis. In this work, we found that $y g a G$ is a chromosomal gene of S. flexneri M90T. YgaG, which is the product of this gene, shares $90 \%$ of identity with LuxS involving in quorum sensing and biofilm formation $[25,26]$. RhlA, RhlB, and RhlR proteins are known to promote the rhamnolipid secretion [27]. The secretion of biosurfactant is correlated with quorum sensing [28].

Pathogenicity in genus Shigella is determined by T3SS that has the ability to secrete a myriad of effector proteins into the target cells [29, 30]. In the absence of cellular contact, the secretory apparatus is not functional [31]; however, some proteins are secreted in leak condition. Cell contact is mimicked using Congo red [11]. Under the Congo red induction condition, all Shigella strains emulsified gasoline and diesel fuels, while the S. flexneri 5a M90T spa40 mutant did not emulsify them anymore. The mutant $S$. flexneri spa40- has no T3SS [11]. The S. flexneri spa40- in a noninducible condition [32] or in a Congo red induction condition does not produce BLM. In addition, by blocking T3SS using benzoic and salicylic acids compounds, we have demonstrated that BLM could not be secreted in extracellular medium. This confirms that BLM is secreted via T3SS. $P$. aeruginosa could secrete BLM in the presence or in the absence of inhibitors. This allows us to postulate that rhamnolipid molecule could use another pathway. An efflux mechanism is the top in $P$. aeruginosa $[10,33]$. This inhibition assay with benzoic acid and salicylic acids showed a perfect correlation between the secretion of the BLM synthesized by Shigella and the inactivation of the type III secretion apparatus.

Regarding the BLM characteristics, precipitation assay such as hydrochloric acid, ammonium sulfate and ethanol allowed postulating that the secreted BLM could have a 


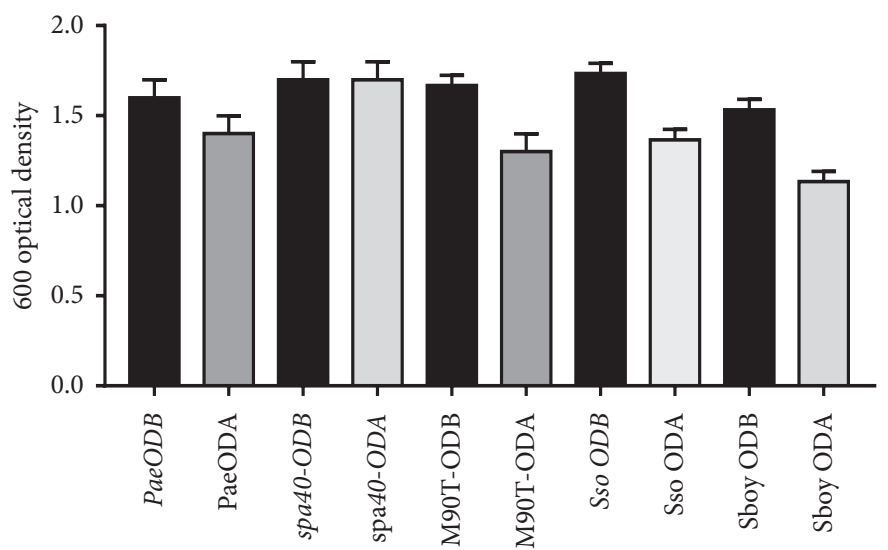

(a)

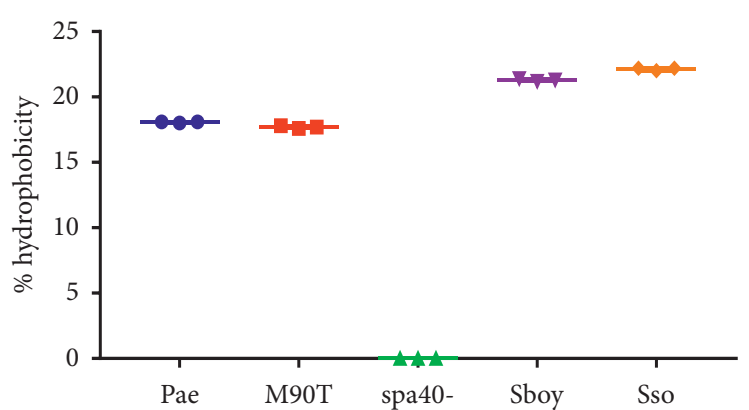

(b)

Figure 4: (a) Shigella's adhesion to hydrocarbon phase of some strains used in this study. ODB: optical density before vortexing; ODA: optical density after vortexing; M90T: Shigella flexneri 5a strain M90T; Sbo: S. boydii; Sso: S. sonnei; spa40-: S. flexneri 5a spa40-; Pae: $P$. aeruginosa used as positive control. (b) Percentage hydrophobicity of Shigella strains.

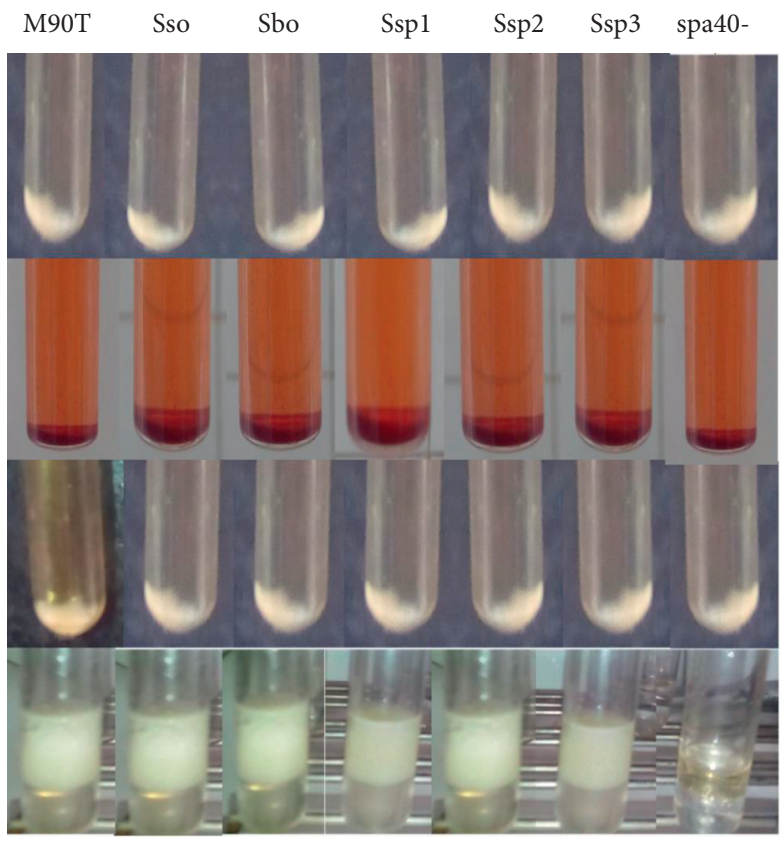

(a)
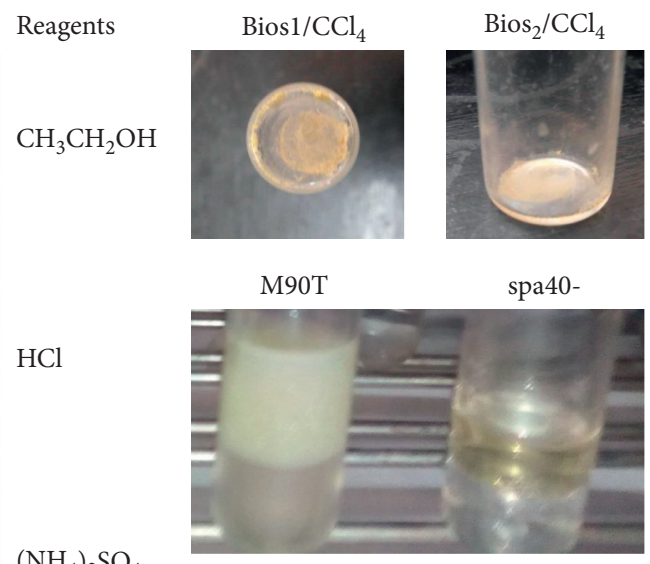

$\left(\mathrm{NH}_{4}\right)_{2} \mathrm{SO}_{4}$

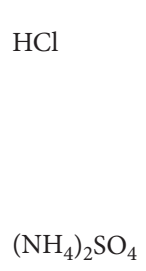

EI24

Figure 5: BLM purified from Shigella sp. TOP: profile obtained after precipitation with ethanol $\left(\mathrm{CH}_{3} \mathrm{CH}{ }_{2} \mathrm{OH}\right)$, hydrochloric acid (HCl), and ammonium sulfate $\left(\left(\mathrm{NH}_{4}\right)_{2} \mathrm{SO}_{4}\right)$. EI24: emulsification index for all strains. S. flexneri M90T, S. sonnei, S. boydii, and Shigella sp.: Ssp1, 2, and 3. Bottom panel: residues obtained after evaporation of chloroform $\left(\mathrm{CCl}_{4}\right)$ (left); emulsification index (EI24) for the extractable biosurfactant-like molecule (right). Bios1 and Bios2: biosurfactant-like molecule residues.

lipopeptide or peptide features. Only peptide or lipopeptide biosurfactants can precipitate at a very low $\mathrm{pH}$ or with ammonium sulphate $[34,35]$. In proteomics studies, the sequential precipitation of ammonium sulfate proteins allows the proteins to be separated by "salting-in" or "saltingout" effect [36], which necessarily leads to the formation of protein aggregates and therefore to their precipitation. The BLM precipitate was able to emulsify gasoline and diesel fuel. Biosurfactants, like rhamnolipid, surfactin, and emulsan, are extractable by organic solvents [14, 37]. In addition, our study showed that the biosurfactant excreted by Shigella sp. is extractable with chloroform with higher efficiency and stability at $40^{\circ} \mathrm{C}$.

BML are known to play several vital roles especially in the microbe's adhesion, bioavailability, desorption, and defense strategy. The most important role of microbial BLM is well reviewed for adhesion of the interfaces in cells-cells interactions [38]. P. aeruginosa is the best example of cell 


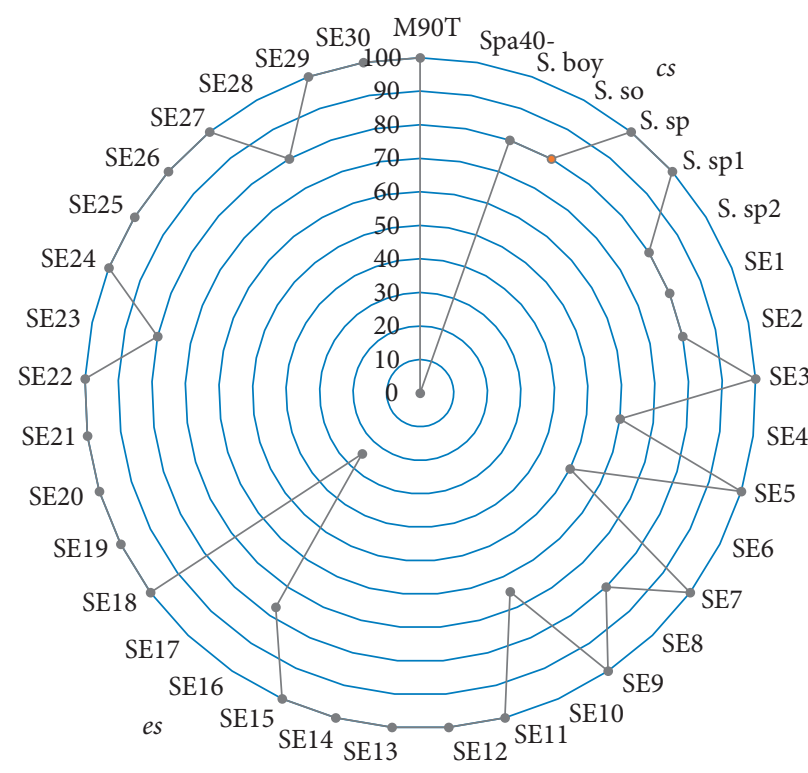

(a)

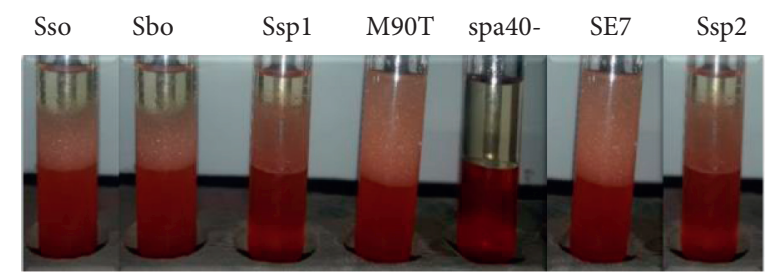

(b)

Figure 6: Emulsification index after Congo red induction. (a) cs: clinical strains; es: environmental strains; Pae: P. aeruginosa used as positive control; M90T: Shigella flexneri 5a strain M90T; spa40-: S. flexneri spa40 mutant; Sbo: S. boydii; Sso: S. sonnei; Ssp, Ssp1, Ssp2: Shigella sp. from clinical strains; SE1 to SE30: Shigella sp. from environmental strains. (b) Emulsification index appearance of some Shigella strains.
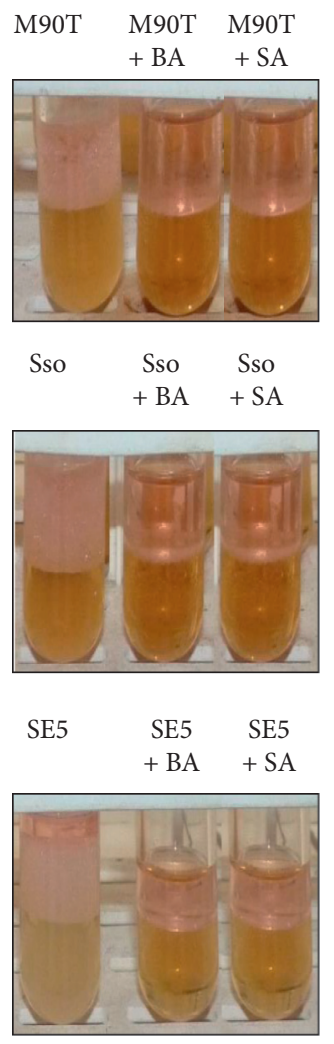

(a)
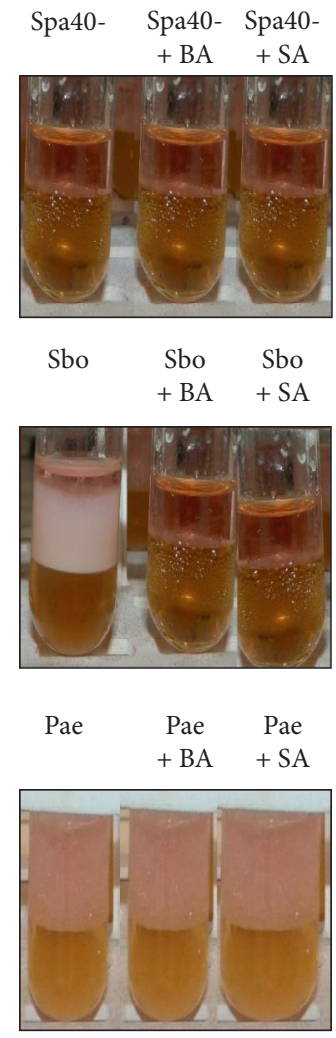

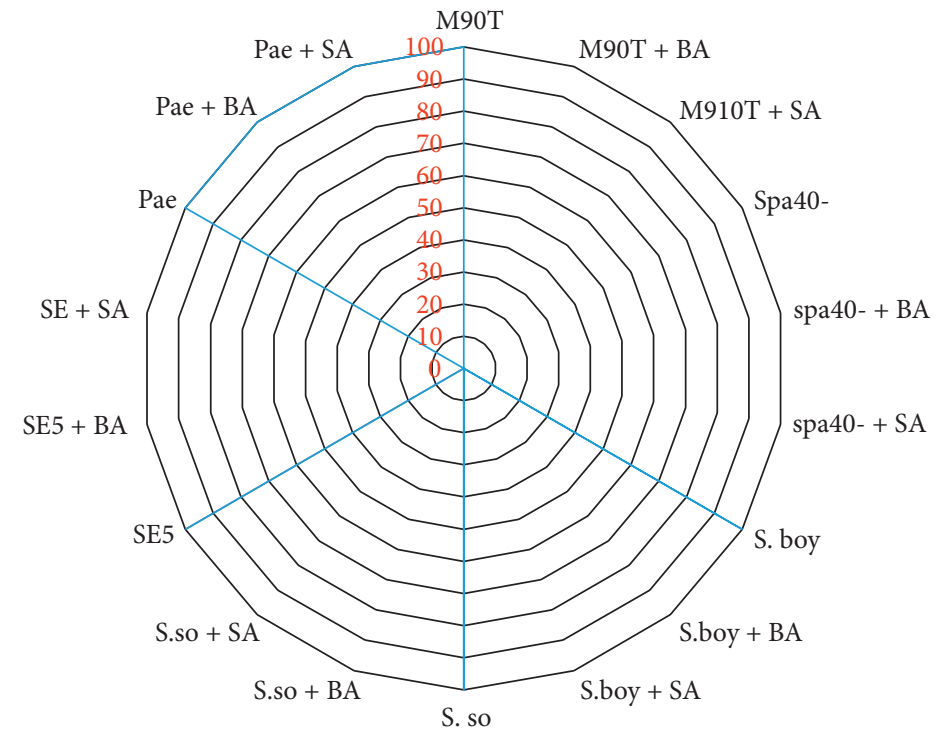

(b)

FIGURE 7: (a) Gasoline emulsifying activity of some Shigella strains used in this study with and without benzoic acid (BA) and salicylic acid (SA). M90T: S. flexneri strain M90T. spa40-: S. flexneri spa40 mutant, Sso: S. sonnei; Sbo: S. boydii; SE: Shigella sp. (environmental strain); Pae: P. aeruginosa. (b) Gasoline emulsifying activity appearances of some Shigella strains tested with and without benzoic acid or salicylic acid. 
surface hydrophobicity which is justified by the presence of cell-bound rhamnolipid [39]. Our new finding showed that, by secreting BLM, Shigella sp. can easily bind to the cell hydrophobic interfaces by interacting with lipid rafts $[30,40-42]$. By binding on cell membrane, BLM allows the reduction of the membrane tension and to help the translocon-like IpaB-C $[43,44]$ and the tip component IpaD $[45,46]$ to be close to the host membrane and automatically inserted inside the cytoplasmic membrane.

Many mechanisms have demonstrated how S. flexneri can disseminate inside epithelial cells [47, 48], helping to escape autophagy phenomenon [49] and to spread inside host cell [50] by using a specific domain of IcsB that interacts with cholesterol [30]. In this work, we showed that $S$. flexneri, $S$. boydii, and $S$. sonnei could spread using the swarming phenomenon. No studies have previously documented the ability of swarming in the mentioned conditions. This efficiently emphasized and amplified the idea that Shigella could be able to use several mechanisms that help spreading from cell to cell by secreting BLM. We are investigating the secretion of BLM inside epithelial cells. Based on our finding, we can propose that Shigella can invade and disseminate inside the epithelial cells using BLM pathways.

\section{Conclusion}

In order to contribute to the understanding of the mechanism of invasion of epithelial cells by Shigella sp., we have first shown that all Shigella strains as well as clinical or environmental strains are able to secrete biosurfactant-like molecules directly in the extracellular medium. Second, we have shown that the secretion of biosurfactants-like molecule depends on type three secretion system (T3SS). Our study suggests that the biosurfactant with lipopeptide or peptide features, stable at $40^{\circ} \mathrm{C}$, could play an outstanding role in Shigella pathogenicity mechanisms including bacteria-host cell interaction, cell metabolism, and cell dissemination. This work contributes to the understanding of genes associated with a couple of components that are able to promote the biosynthesis, regulation, and secretion of BLM. Knocking out Shigella with couple genes encoded effector proteins such as IpaB, IpaC, and IpaD could orient investigation. In the continuation of our study experiments including MALDI-TOF and HPLC are in its ways to more biochemically characterize BLM. .

\section{Data Availability}

The Excel sheets including the data used to support the findings of this study are available from the corresponding author upon request.

\section{Conflicts of Interest}

The authors declare that there are no conflicts of interest.

\section{Acknowledgments}

The authors are grateful to Prof. Eric Déziel (Centre Armand-Frappier Santé Biotechnologie) for his deep precious and wise advice, Prof. Anne Botteaux (Free University of Brussels) for scientific discussion, and Dr. Armel Ibala Zamba for their continuous encouragements and for helpful data analysis before publication and to Ms. Dalila Lakhloufi, Mr. Loic Marly Djesone Bantsimba Malonga and Mr. Chrislen Kapende for providing Shigella sp. strains.

\section{References}

[1] L. Karen, M. S. R. Kotloff, J. A. Platts-Mills, P. Pavlinac, and K. M. Z. Anita, "Shigellosis," Lancet, vol. 391, pp. 801-812, 2018.

[2] K. L. Kotloff, J. P. Nataro, W. C. Blackwelder et al., "Burden and aetiology of diarrhoeal disease in infants and young children in developing countries (the global enteric multicenter study, GEMS): a prospective, case-control study," Lancet, vol. 382, no. 9888, pp. 209-222, 2013.

[3] K. L. Kotloff, J. P. Winickoff, B. Ivanoff et al., "Global burden of Shigella infections: implications for vaccine development and implementation of control strategies," Bulletin of the World Health Organization, vol. 77, no. 8, pp. 651-666, 1999.

[4] T. L. Hale, "Genetic basis of virulence in Shigella species," Microbiological Reviews, vol. 55, no. 2, pp. 206-224, 1991.

[5] C. A. Kayath, A. Ibala Zamba, J. Goma-Tchimbakala et al., "Microbiota landscape of gut system of guppy fish (Poecilia reticulata) plays an outstanding role in adaptation mechanisms," International Journal of Microbiology, vol. 2019, Article ID 3590584, 10 pages, 2019.

[6] R. Marchant and I. M. Banat, "Microbial biosurfactants: challenges and opportunities for future exploitation," Trends in Biotechnology, vol. 30, no. 11, pp. 558-565, 2012.

[7] I. M. Banat, R. S. Makkar, and S. S. Cameotra, "Potential commercial applications of microbial surfactants," Applied Microbiology and Biotechnology, vol. 53, no. 5, pp. 495-508, 2000.

[8] A. M. Abdel-Mawgoud, F. Lépine, and E. Déziel, "Rhamnolipids: diversity of structures, microbial origins and roles," Applied Microbiology and Biotechnology, vol. 86, no. 5, pp. 1323-1336, 2010.

[9] M. Rosenberg, D. Gutnick, and E. Rosenberg, "Adherence of bacteria to hydrocarbons: a simple method for measuring cellsurface hydrophobicity," FEMS Microbiology Letters, vol. 9, no. 1, pp. 29-33, 1980.

[10] M. M. Usman, A. Dadrasnia, K. T. Lim, A. F. Mahmud, and S. Ismail, "Application of biosurfactants in environmental biotechnology, remediation of oil and heavy metal," AIMS Bioengineering, vol. 3, no. 3, pp. 289-304, 2016.

[11] A. Botteaux, M. Sani, C. A. Kayath, E. J. Boekema, and A. Allaoui, "Spa32 interaction with the inner-membrane Spa40 component of the type III secretion system of Shigella flexneriis required for the control of the needle length by a molecular tape measure mechanism," Molecular Microbiology, vol. 70, no. 6, pp. 1515-1528, 2008.

[12] A. Botteaux, M. Sani, C. A. Kayath, E. J. Boekema, and A. Allaoui, "Spa32 interaction with the inner-membrane Spa40 component of the type III secretion system of Shigella flexneriis required for the control of the needle length by a molecular tape measure mechanism," Molecular Microbiology, vol. 70, no. 6, pp. 1515-1528, 2008.

[13] A. Zdarta, W. Smułek, A. Trzcińska, Z. Cybulski, and E. Kaczorek, "Properties and potential application of efficient biosurfactant produced by Pseudomonas sp. KZ1 strain," Journal of Environmental Science and Health, Part A, vol. 54, no. 2, pp. 110-117, 2019. 
[14] S. J. Varjani and V. N. Upasani, "Critical review on biosurfactant analysis, purification and characterization using rhamnolipid as a model biosurfactant," Bioresource Technology, vol. 232, pp. 389-397, 2017.

[15] E. M. Rossi, L. Beilke, M. Kochhann, D. H. Sarzi, and E. C. Tondo, "Biosurfactant produced by Salmonella enteritidis SE86 can increase adherence and resistance to sanitizers on lettuce leaves (Lactuca sativa L., cichoraceae)," Frontiers in Microbiology, vol. 7, no. 9, 2016.

[16] G. Chen, M. Qiao, H. Zhang, and H. Zhu, "Bacterial desorption in water-saturated porous media in the presence of rhamnolipid biosurfactant," Research in Microbiology, vol. 155 , no. 8 , pp. $655-661,2004$.

[17] H. W. C. Araujo, R. F. S. Andrade, D. Montero-Rodriguez, D. Rubio-Ribeaux, C. A. Alves da Silva, and G. M. CamposTakaki, "Sustainable biosurfactant produced by Serratia marcescens UCP 1549 and its suitability for agricultural and marine bioremediation applications," Microbial Cell Factories, vol. 18, no. 1, p. 2, 2019.

[18] D. G. Cooper and B. G. Goldenberg, "Surface-active agents from two Bacillus species," Applied and Environmental Microbiology, vol. 53, no. 2, pp. 224-229, 1987.

[19] S. K. Satpute, A. G. Banpurkar, P. K. Dhakephalkar, I. M. Banat, and B. A. Chopade, "Methods for investigating biosurfactants and bioemulsifiers: a review," Critical Reviews in Biotechnology, vol. 30, no. 2, pp. 127-144, 2010.

[20] M. D. Kaya-Ongoto, C. A. Kayath, A. B. V. Mbozo et al., "Prime enzymatic exocellular background of Lysinibacillus louembei," Advances in Microbiology, vol. 10, no. 3, pp. 95-109, 2020.

[21] E. Deziel, F. Lepine, S. Milot, and R. Villemur, "rhlA is required for the production of a novel biosurfactant promoting swarming motility in Pseudomonas aeruginosa: 3-(3hydroxyalkanoyloxy)alkanoic acids (HAAs), the precursors of rhamnolipids," Microbiology, vol. 149, no. Pt 8, pp. 2005-2013, 2003.

[22] R. Belas and R. Suvanasuthi, "The ability of Proteus mirabilis to sense surfaces and regulate virulence gene expression involves FliL, a flagellar basal body protein," Journal of Bacteriology, vol. 187, no. 19, pp. 6789-6803, 2005.

[23] N. R. Williamson, P. C. Fineran, W. Ogawa, L. R. Woodley, and G. P. C. Salmond, "Integrated regulation involving quorum sensing, a two-component system, a GGDEF/EAL domain protein and a post-transcriptional regulator controls swarming and RhlA-dependent surfactant biosynthesis in Serratia," Environmental Microbiology, vol. 10, no. 5, pp. 1202-1217, 2008.

[24] M. T. Butler, Q. Wang, and R. M. Harshey, "Cell density and mobility protect swarming bacteria against antibiotics," Proceedings of the National Academy of Sciences, vol. 107, no. 8, pp. 3776-3781, 2010.

[25] W. A. Day Jr. and A. T. Maurelli, "Shigella flexneri LuxS quorum-sensing system modulates virB expression but is not essential for virulence," Infection and Immunity, vol. 69, no. 1, pp. 15-23, 2001.

[26] P. H. Brito, E. P. C. Rocha, K. B. Xavier, and I. Gordo, "Natural genome diversity of AI-2 quorum sensing in Escherichia coli: conserved signal production but labile signal reception," Genome Biology and Evolution, vol. 5, no. 1, pp. 16-30, 2013.

[27] G. Soberon-Chavez, M. Aguirre-Ramirez, and R. Sanchez, "The Pseudomonas aeruginosa RhlA enzyme is involved in rhamnolipid and polyhydroxyalkanoate production," Journal of Industrial Microbiology \& Biotechnology, vol. 32, no. 11-12, pp. 675-677, 2005.
[28] J. P. Pearson, E. C. Pesci, and B. H. Iglewski, "Roles of Pseudomonas aeruginosa las and rhl quorum-sensing systems in control of elastase and rhamnolipid biosynthesis genes," Journal of Bacteriology, vol. 179, no. 18, pp. 5756-5767, 1997.

[29] P. J. Sansonetti, J. Arondel, J. R. Cantey, M. C. Prévost, and M. Huerre, "Infection of rabbit Peyer's patches by Shigella flexneri: effect of adhesive or invasive bacterial phenotypes on follicle-associated epithelium," Infection and Immunity, vol. 64, no. 7, pp. 2752-2764, 1996.

[30] C. A. Kayath, S. Hussey, N. El hajjami, K. Nagra, D. Philpott, and A. Allaoui, "Escape of intracellular Shigella from autophagy requires binding to cholesterol through the type III effector, IcsB," Microbes and Infection, vol. 12, no. 12-13, pp. 956-966, 2010.

[31] A. Anantharajah, M.-P. Mingeot-Leclercq, and F. Van Bambeke, "Targeting the type three secretion system in Pseudomonas aeruginosa," Trends in Pharmacological Sciences Cell Press, vol. 37, no. 9, pp. 734-749, 2016.

[32] C. Parsot, "Shigella type III secretion effectors: how, where, when, for what purposes?" Current Opinion in Microbiology, vol. 12, no. 1, pp. 110-116, 2009.

[33] U. A. Ochsner and J. Reiser, "Autoinducer-mediated regulation of rhamnolipid biosurfactant synthesis in Pseudomonas aeruginosa," Proceedings of the National Academy of Sciences, vol. 92, no. 14, pp. 6424-6428, 1995.

[34] J. Vater, B. Kablitz, C. Wilde, P. Franke, N. Mehta, and S. S. Cameotra, "Matrix-assisted laser desorption ionizationtime of flight mass spectrometry of lipopeptide biosurfactants in whole cells and culture filtrates of Bacillus subtilis C-1 isolated from petroleum sludge," Applied and Environmental Microbiology, vol. 68, no. 12, pp. 6210-6219, 2002.

[35] I. M. Banat, T. J. Smyth, A. Perfumo, S. McClean, and R. Marchant, "Isolation and analysis of lipopeptides and high molecular weight biosurfactants," Handbook of Hydrocarbon and Lipid Microbiology, Springer, Berlin, Germany, pp. 3687-3704, 2010.

[36] A. A. Green and W. L. Hughes, "Protein solubility on the basis of solubility in aqueous solutions of salts and organic solvents," Methods in Enzymology, Elsevier, vol. 1, pp. 67-90, , Amsterdam, Netherlands, 1995.

[37] P. Singh, Y. Patil, and V. Rale, "Biosurfactant production: emerging trends and promising strategies," Journal of Applied Microbiology, vol. 126, no. 1, pp. 2-13, 2019.

[38] S. S. Cameotra, R. S. Makkar, J. Kaur, and S. K. Mehta, "Synthesis of biosurfactants and their advantages to microorganisms and mankind," Advances in Experimental Medicine and Biology, vol. 672, pp. 261-280, 2010.

[39] Y. Zhang and R. M. Miller, "Effect of a Pseudomonas rhamnolipid biosurfactant on cell hydrophobicity and biodegradation of octadecane," Applied and Environmental Microbiology, vol. 60, no. 6, pp. 2101-2106, 1994.

[40] F. Lafont and F. G. van der Goot, "Bacterial invasion via lipid rafts," Cellular Microbiology, vol. 7, no. 5, pp. 613-620, 2005.

[41] M. Edidin, "The state of lipid rafts: from model membranes to cells," Annual Review of Biophysics and Biomolecular Structure, vol. 32, no. 1, pp. 257-283, 2003.

[42] R. D. Hayward, R. J. Cain, E. J. McGhie, N. Phillips, M. J. Garner, and V. Koronakis, "Cholesterol binding by the bacterial type III translocon is essential for virulence effector delivery into mammalian cells," Molecular Microbiology, vol. 56, no. 3, pp. 590-603, 2005.

[43] S.-C. Yang, C.-F. Hung, I. A. Aljuffali, and J.-Y. Fang, "The roles of the virulence factor IpaB in Shigella spp. in the escape 
from immune cells and invasion of epithelial cells," Microbiological Research, vol. 181, pp. 43-51, 2015.

[44] A. Blocker, P. Gounon, E. Larquet et al., "The tripartite type III secreton of Shigella flexneri inserts IpaB and IpaC into host membranes," Journal of Cell Biology, vol. 147, no. 3, pp. 683-693, 1999.

[45] L. Schiavolin, A. Meghraoui, Y. Cherradi, L. Biskri, A. Botteaux, and A. Allaoui, "Functional insights into the Shigella type III needle tip IpaD in secretion control and cell contact," Molecular Microbiology, vol. 88, no. 2, pp. 268-282, 2013.

[46] M. Sani, A. Botteaux, C. Parsot, P. Sansonetti, E. J. Boekema, and A. Allaoui, "IpaD is localized at the tip of the Shigella flexneri type III secretion apparatus," Biochimica et Biophysica Acta (BBA) - General Subjects, vol. 1770, no. 2, pp. 307-311, 2007.

[47] H. Agaisse, "Molecular and cellular mechanisms of Shigella flexneri dissemination," Front Cell Infect Microbiol, vol. 6, p. $29,2016$.

[48] B. J. Koestler, C. R. Fisher, and S. M. Payne, "Formate promotes Shigella intercellular spread and virulence gene expression," mBio, vol. 9, no. 5, 2018.

[49] M. Ogawa, T. Suzuki, I. Tatsuno, H. Abe, and C. Sasakawa, "IcsB, secreted via the type III secretion system, is chaperoned by IpgA and required at the post-invasion stage of Shigella pathogenicity," Molecular Microbiology, vol. 48, no. 4, pp. 913-931, 2003.

[50] A. Allaoui, J. Mounier, M.-C. Prévost, P. J. Sansonetti, and C. Parsot, "icsB: a Shigella flexneri virulence gene necessary for the lysis of protrusions during intercellular spread," Molecular Microbiology, vol. 6, no. 12, pp. 1605-1616, 1992. 